\title{
Dynamics accompanying polarization switching in vertical-cavity surface-emitting lasers.
}

\author{
Martin Virte $^{a, b}$ and Krassimir Panajotov ${ }^{b, c}$ and Marc Sciamanna ${ }^{a}$ \\ ${ }^{a}$ Supélec, OPTEL Research Group, Laboratoire Matériaux Optiques, Photonique et Systèmes \\ (LMOPS) EA-4423, 2 Rue Edouard Belin, F-57070 Metz, France \\ ${ }^{b}$ Department of Applied Physics and Photonics (IR-TONA), Vrije Universiteit Brussels, \\ Pleinlaan 2, 1050 Brussels, Belgium \\ ${ }^{c}$ Institute of Solid State Physics, 72 Tzarigradsko Chaussee Blvd., 1784 Sofia, Bulgaria
}

\begin{abstract}
Experiments on quantum well and recently quantum dot VCSELs have shown that the increase of injection current may lead to a transition from a linearly polarized light emission at threshold to a region of nonlinear dynamics (self-pulsing) accompanying polarization switching between either two orthogonal linearly polarized states or non-orthogonal elliptically polarized states. The dynamics occurs on a nanosecond time-scale and relates either to the birefringence induced frequency splitting or to the relaxation oscillation frequency.

In this contribution, we bring new light into the bifurcation mechanisms explaining the occurrence of deterministic self-pulsing accompanying polarization switching. We demonstrate theoretically that depending on the laser parameters, different polarization switching scenarios may be observed with self-pulsing dynamics at a dominant time-scale related to either linear cavity birefringence or relaxation oscillation, and with additional period doubling or quasiperiodicity. Our work therefore not only reconciles previous experiments with different conclusions on dynamical states, but also provides an improved understanding of the bifurcations underlying the commonly used spin flip model for VCSEL - hence motivating new in depth experiments of polarization dynamics at nanosecond time scale.
\end{abstract}

Keywords: Vertical-cavity surface-emitting laser, Polarization, Quantum dot lasers, nonlinear dynamics

\section{INTRODUCTION}

Vertical-cavity surface-emitting lasers (VCSELs) are an important step forward for optical communications as they exhibits many advantages over Edge-emitting lasers (EELs) both in terms of performance and production efficiency. However in these structures the polarization selection is much weaker than in EELs, thus many experiments have reported cases of polarization instabilities and even a particularly striking event called polarization switching (PS) ${ }^{1-8}$ Although a VCSEL generally starts emitting in a linearly polarized (LP) mode, an increase of the injection current can induce a switching to the orthogonal LP mode. ${ }^{5,6}$ As a result of cavity birefringence these two modes are frequency detuned, we refer to them as the low (LF) and high (HF) frequency linear polarization. A switching from HF to LF (resp. LF to HF) is identified as a type I PS (resp. type II PS), but cases where the laser experiences two PSs as the current is increased (type I then type II) have also been observed. ${ }^{6}$ Typically PS is accompanied by noise driven bistable mode hopping, ${ }^{9}$ but two experiments have identified more complex dynamical transition accompanying PSs. Thus it appears that the increase of current can lead to elliptically polarized states and complex dynamical self-pulsing occurring at nanosecond time-scale. ${ }^{5-8}$ The frequency of these dynamics appeared to relate either to the birefringence induced frequency ${ }^{6}$ or to the relaxation oscillation frequency. ${ }^{8}$ The bifurcations underlying these dynamical transitions and what determines the frequencies of the self-pulsing dynamics remain issues to be clarified.

Further author information: (Send correspondence to M.V.)

E-mail: martin.virte@supelec.fr 
A dynamical approach of these polarization instabilities in VCSEL has been proposed by San Miguel et al, ${ }^{10,11}$ the so-called San Miguel-Feng-Moloney (SFM) or spin-flip model. A four-level model is considered, accounting for two separated processes for the left $(-)$ and right $(+)$ circularly polarized emission with two separated carrier reservoir but coupled through the complex spin-flip processes equilibrating the carrier population. SFM has later been extended to include cavity anisotropies. ${ }^{11-13}$ The SFM steady-states have been extensively studied by means of asymptotic methods or direct numerical integration ${ }^{11-17}$ and it appears that SFM allows for linearly and elliptically polarized states. The stability analysis of these steady-states revealed that both type I and type II PSs are possible in the framework of SFM, the inclusion of additional parameters (e.g. gain saturation) allowing to reproduce more complex sequences like double PS. ${ }^{16,18,19}$ Despite these analysis, there is, to our best knowledge, no investigation of the higher order bifurcations that may lead to complex nonlinear dynamics. In particular there is no study of the frequencies of the polarization dynamics and how they may depend on the laser parameters.

In this contribution, by means of direct numerical integration and advance continuation techniques using the DDE-BIFTOOL package, ${ }^{20}$ we provide a valuable insight of the bifurcation scenarios leading to complex nonlinear dynamics in the framework of SFM. Continuation techniques allow to follow numerical solution in the phase-space regardless of their stability and therefore to reveal the underlying mechanism and bifurcation sequences creating the dynamical states. Of particular interest is the Hopf bifurcations analysis which gives the fundamental frequency of the emerging periodic solution. Therefore we make an in-depth bifurcation analysis of the scenario leading to self-pulsating dynamics and we demonstrate that the frequency of these dynamics is in fact a bifurcation problem: depending on the parameters, the frequency can be either close to the birefringence induced frequency or to the relaxation oscillation frequency. Thus our work reconciles both experiments ${ }^{6,8}$ but also brings unprecedented new details to our theoretical understanding of SFM.

This paper is organized as follow: in section 2 we present in detail the rate equation of SFM. In section 3 we provide the global bifurcation analysis focusing on dynamical states and in section 4 we make an in-depth analysis of a type II polarization switching. In section 5 we demonstrate the good agreement between our type II PS bifurcation sequence and the experimental observations of Sondermann et al. ${ }^{6}$ and we present additional results for a scenario close to the one reported by Olejniczak et al. ${ }^{8}$ Finally we discuss and summarize our results in section 6 .

\section{RATE EQUATION OF THE SYSTEM: THE SAN MIGUEL-FENG-MOLONEY APPROACH}

\subsection{Rate equation of the system}

The San Miguel-Feng-Moloney model (SFM) or spin-flip model described by San Miguel et al, ${ }^{10,11}$ considers two slowly varying enveloppes for the left $E_{-}=R_{-} e^{i \psi_{-}}$and right $E_{+}=R_{+} e^{i \psi_{+}}$circularly polarized emissions with two distinct carrier populations $D_{-}$and $D_{+}$. Yet the population carrier is described by $N=D_{+}+D_{-}$the total carrier population and $n=D_{+}-D_{-}$the population difference. For simplicity and to allow for a numerical treatment, we consider the phase difference $\Phi=\psi_{+}-\psi_{-}$, see. ${ }^{14}$ The SFM equation set can be written as follows:

$$
\begin{aligned}
& \frac{d R_{+}}{d t}=\kappa(N+n-1) R_{+}-\left(\gamma_{a} \cos (\Phi)+\gamma_{p} \sin (\Phi)\right) R_{-} \\
& \frac{d R_{-}}{d t}=\kappa(N-n-1) R_{-}-\left(\gamma_{a} \cos (\Phi)-\gamma_{p} \sin (\Phi)\right) R_{+} \\
& \frac{d \Phi}{d t}=2 \kappa \alpha n-\left(\frac{R_{-}}{R_{+}}-\frac{R_{+}}{R_{-}}\right) \gamma_{p} \cos (\Phi)+\left(\frac{R_{+}}{R_{-}}+\frac{R_{+}}{R_{-}}\right) \gamma_{a} \sin (\Phi) \\
& \frac{d N}{d t}=-\gamma\left[N-\mu+(N+n) R_{+}^{2}+(N-n) R_{-}^{2}\right] \\
& \frac{d n}{d t}=-\gamma_{s} n-\gamma\left[(N+n) R_{+}^{2}-(N-n) R_{-}^{2}\right]
\end{aligned}
$$


$\kappa$ is the decay rate of the electric field in the cavity, $\alpha$ is the linewidth enhancement factor, $\mu$ is the normalized injection current. $\gamma$ is the decay rate of the total carrier number and $\gamma_{s}$ is the decay rate of the spin-flip relaxation processes that equilibrates the spin orientation of both carrier reservoirs. The phase and amplitude anisotropies are noted $\gamma_{p}$ and $\gamma_{a}$. We applied a chosen frequency shift of $\omega_{0}=\kappa \alpha$ to the original set of equation which lead to a zero emission frequency for $E_{ \pm}$at threshold for $\gamma_{a}=0$ and $\gamma_{p}=0$; thus $\omega_{0}$ is our reference frequency. In this contribution, unless stated otherwise, we will use the following parameter values: $\gamma_{a}=-0.7 n s^{-1}, \gamma_{s}=100 n s^{-1}$, $\gamma=1, \gamma_{p} \in[0,100] n s^{-1}, \kappa=600 n s^{-1}, \alpha=3$ et $\mu \in[1,10]$. The system is symmetric in terms of birefringence $\gamma_{p}$ for the right and left circularly polarized light emissions, hence we will only use $\gamma_{p}>0$.

\subsection{SFM steady-states}

In the frame of SFM, we find two linearly polarized (LP) steady-states: X and Y-LP, standing respectively for the low and high frequency modes for $\gamma_{p}>0$ : their respective frequencies are $\omega_{X}=-\gamma_{p}+\gamma_{a} \alpha$ and $\omega_{Y}=\gamma_{p}-\gamma_{a} \alpha$. The splitting frequency between the two LP states is therefore given by $2 \pi F_{s p l i t}=2 \gamma_{p}-2 \alpha \gamma_{p}$, the other characteristic frequency of the system being the relaxation oscillation frequency given by $2 \pi F_{R O}=\sqrt{2 \kappa(\mu-1)}$. The two LP steady-states are given by:

$$
R_{ \pm}=\sqrt{\frac{\mu-N_{0}}{2 N_{0}}}, \quad n_{0}=0, \quad N_{0, X}=1+\frac{\gamma_{a}}{\kappa}, \quad \Phi_{X}=0, \quad N_{0, Y}=1-\frac{\gamma_{a}}{\kappa}, \quad \text { and } \quad \Phi_{Y}=\pi
$$

The threshold for the two LP steady-states is given by $\mu=N_{0}$, hence it depends on the amplitude anisotropy $\gamma_{a}$. For $\gamma_{a}<0$ the laser should starts lasing on the X-LP mode which has the lower threshold; the problem is inverted for $\gamma_{a}>0$.

SFM also includes two elliptically polarized (EP) steady-states which cannot be described analytically with $\gamma_{p} \neq 0$ and $\gamma_{a} \neq 0$ and which are created by a pitchfork bifurcation on the X-LP branch; the two states are symmetrical with respect to the $\mathrm{X}$ axis.

\section{GLOBAL BIFURCATION ANALYSIS}

By using advance continuation techniques with the DDE-BIFTOOL package ${ }^{20}$ we perform a global bifurcation analysis of the SFM model. We follow steady-state solution and plot their stability informations in the plane of parameter: injection current $\mu$ versus phase anisotropy $\gamma_{p}$, see figure 1, panel (a). The Y-LP is stable on the left of the red dash-dotted curve, the curve stands for a hopf bifurcation destabilizing the Y-LP steady-state into a periodic solution. The X-LP is stable under the black dashed curve which represents a pitchfork bifurcation destabilizing the X-LP state and creating the two symmetrical EP states. The blue curve is a hopf bifurcation destabilizing the EP states; hence these states are stable only between the black and the blue curves. Beyond the blue curve and on the right on the red dash-dotted curve no stable steady-states exist. Thus we are particularly interested in the Hopf bifurcations which lead to self-pulsating dynamics at a frequency close to the Hopf frequency. Figure 1-(b) gives the evolution of the Hopf frequency along the Hopf curve in (a); the panel (c) gives the normalization with respect to the relaxation oscillation frequency.

We identify as much as nine different bifurcation scenarios, however the scenarios have much in common. On figure 1-(left), i.e. scenarios 1 to 5, the laser start lasing on the Y-LP mode; on the right side of figure 1 all scenarios start lasing in X-LP mode.

- case 1 - blue area (color online) of figure 1-(left): the laser starts emitting in Y-LP and this state remains stable for all injection current value; no switching appears in this case. If somehow the laser starts lasing in X-LP (e.g. due to a large amount of noise ${ }^{6}$ ), the scenario would be similar to case 6 .

- case 2 - gray are (color online) of figure 1-(left): the laser emits in Y-LP but at a critical value of the injection current a hopf bifurcation occur. As no stable-state is stable beyond this value, the laser experiences complex dynamics. 

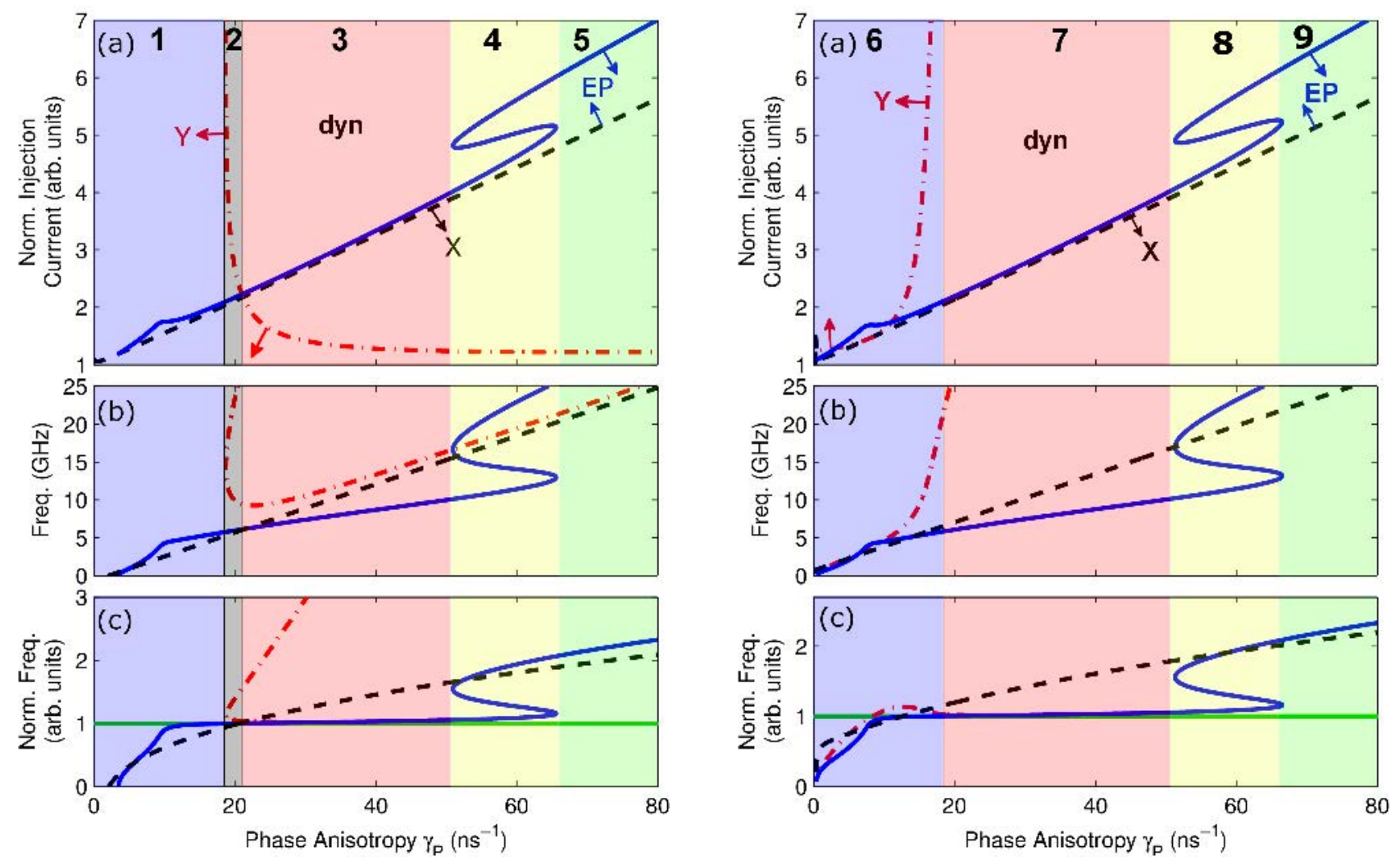

Figure 1. (color online)(a) Stability diagram in the (phase anisotropy, injection current) plane of the linearly and elliptically polarized steady-states for a positive amplitude anisotropy $\gamma_{a}=0.7 \mathrm{~ns}^{-1}$ on the left and for a negative amplitude anisotropy $\left(\gamma_{a}=-0.7 n s^{-1}\right)$ on the right. The red dash-dotted curve is the Y-LP hopf bifurcation, the black dashed curve is the X-LP pitchfork bifurcation and the blue solid curve is the EP hopf bifurcation. (b) Frequencies associated with hopf bifurcation, the legend is identical to (a) except for the dashed black curve which gives the birefringence induced frequency splitting. (c) is the same as (b) but normalized by the ROF given by the green horinzontal solid line.

- case 3 - red area (color online) of figure 1-(left): the laser starts emitting in Y-LP, but a subcritical hopf bifurcation quickly occurs creating a type I switching; no dynamical transition appears. Thus the laser emits in X-LP until a pitchfork bifurcation occur destabilizing the X-LP while creating the two EP states. But the elliptically polarized states are quickly destabilized by a Hopf bifurcation creating self-pulsating dynamics at the relaxation oscillation frequency. After that a sequence of bifurcation not displayed in figure 1 leads the system to a more complex nonlinear dynamic.

- case 4 - yellow area (color online) of figure 1-(left): similar to case 3, but with a region of bistability between periodic solutions and EP states; this scenario has been investigated in detail in. ${ }^{17}$

- case 5 - green area (color online) of figure 1-(left): similar to case 3, but the self-pulsing appears at a much higher frequency compared to the relaxation oscillation frequency; It seems somehow related to the birefringence induced frequency but with a relatively large offset.

- case 6 - blue area (color online) of figure 1-(right): The laser starts on the X-LP mode and as the injection current is increased a pitchfork bifurcation occur, destabilizing the X-LP and creating the two EP states. However a Hopf bifurcation quickly appears destabilizing the EP states and leading the system to a region of dynamics. The frequency of the self-pulsing relates either to the birefringence induced frequency for small phase anisotropy or to the relaxation oscillation frequency for higher value of $\gamma_{p}$. After a complex dynamical transition the system finally reaches the stable Y-LP state thus describing a complete type II polarization switching. For decreasing injection current we find a totally different story as the laser emits 
in Y-LP until a Hopf bifurcation occurs, destabilizing the LP state and leading the system to one of the EP state, then the X-LP state after the pitchfork bifurcation occurred.

- case 7, 8 \& 9 - red, yellow \& green area (color online) of figure 1-(right): these scenarios are very similar to cases $3,4 \& 5$ except that the laser starts lasing on X-LP mode, hence suppressing the first type I PS of the sequence.

With this bifurcation analysis we demonstrate that the frequency of self-pulsating dynamic accompanying a polarization switching is in fact a bifurcation problem: the frequency cannot be linked directly to $F_{\text {split }}$ or $F_{R O}$ but can relate to one or the other frequency depending on the parameters. Therefore, in the framework of SFM, we reconcile both experimental observations of Sondermann et al. ${ }^{6}$ and Olejniczak et al. ${ }^{8}$

\section{NONLINEAR DYNAMICS IN A TYPE II POLARIZATION SWITCHING}

In this section we make an in-depth investigation of a type II polarization switching corresponding to case 6 described in the previous section. We use the following parameters $\gamma_{a}=-0.7$ and $\gamma_{p}=4$, thus we find a complete scenario of type II switching by varying the normalized injection current in $\mu \in[1,2]$. In figure 3 we give the evolution of the fractional polarization (FP) (a), the instantaneous ellipticity (IE) (b), the averaged power in the linearly polarized modes $\mathrm{X}$ and $\mathrm{Y}$ (c) and a polarization resolved bifurcation diagram (d) when increasing the injection current. We show in figure 3 Fast Fourrier Transform (FFT) optical spectra, phase space projection in the plane $\left(\operatorname{Re}\left(E_{X}\right), \operatorname{Re}\left(E_{Y}\right)\right)$ and intensity time-traces for samples at six different currents. Finally we compare these results with those obtained by continuation using the DDE-BIFTOOL package in figure 2 where we plot in panel (a) the maximum and minimum of $A=R_{+}^{2} /(\mu-1)$ versus the normalized injection current $\mu$. Although the representation of figure 2-(a) seems complex, the two linearly polarized states correspond to the same line $A=1 / 2$ (but with different phases) which allow us to identify easily the other states. Both EP are given (blue line) but only one periodic solution emerging from the Hopf bifurcation on the EP branch is displayed (green line). In figure 2-(b) we plot the frequency of the periodic solutions versus the current $\mu$; we also add the birefringence induced splitting frequency $F_{\text {split }}$ (black dashed curve) and the relaxation oscillation frequency $F_{R O}$ (red dashed curve). As we can see we find a good agreement between direct numerical integration results

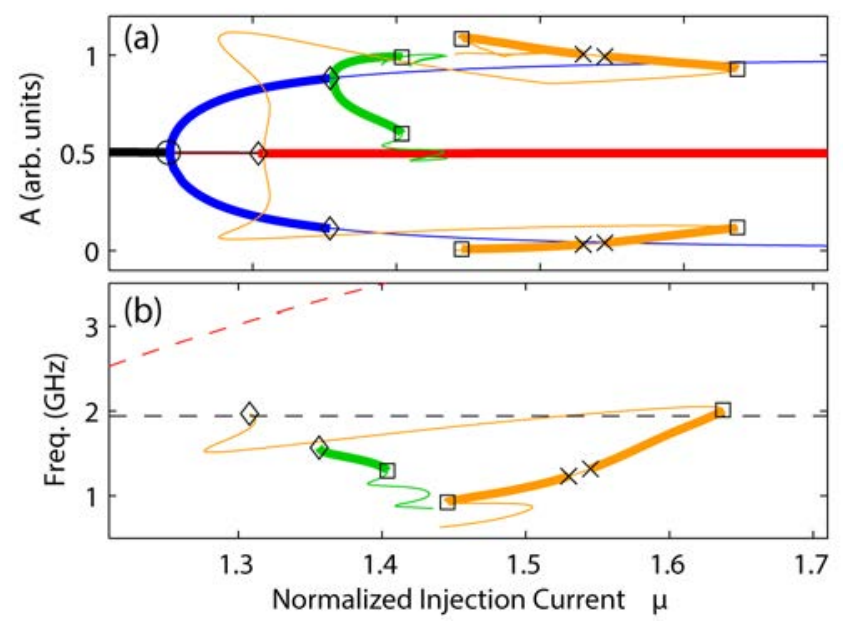

Figure 2. (color online) Continuation results for a type II switching corresponding to case 6 described in sec. 3: the stable (unstable) part of the branches are in thick (thin) lines. (a) Maximum and minimum of $A=R_{+}^{2} /(\mu-1)$ versus normalized injection current (b) Frequency of the periodic solutions versus normalized injection current. The X and Y-LPs are in black and red respectively and the EP states are in blue. The periodic solution created on the EP states is in green (a similar branch is created at the lower hopf bifurcation but not represented) while the one created on the Y-LP state is in orange. Diamonds are hopf, squares are saddle-node and crosses are period doubling bifurcations. On diagram (b) the black dashed line gives $F_{\text {split }}$ and the red one $F_{R O}$ which is not displayed for $\mu>1.4$. 

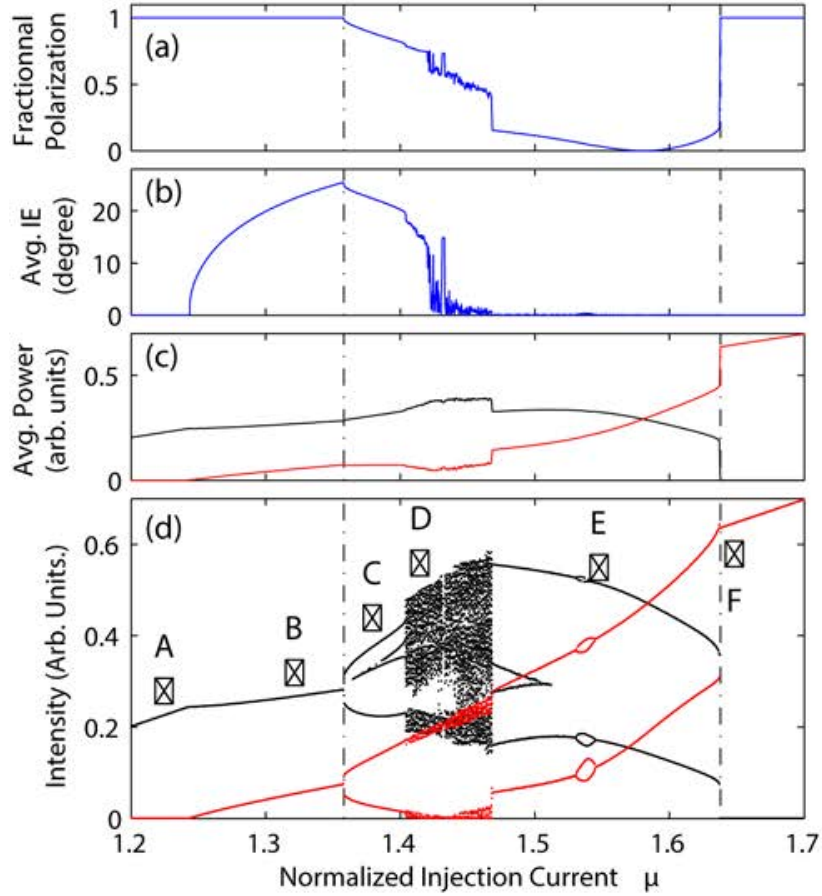

Figure 3. (color online) Direct numerical simulation for a type II switching corresponding to case 6 described in sec. 3: (a) fractional polarization, (b) Averaged Instantaneous Ellipticity, (c) average output power on the X-axis (black) and Y-axis (red) of polarization, (d) bifurcation diagram of the output power on the $\mathrm{X}$-axis (black) and the $\mathrm{Y}$-axis (red) polarization, i.e extrema of the corresponding timeseries. The two vertical dashed line are the limits of the dynamical behavior. The labeled arrows point to the position of the time-series detailed in figure 4 .
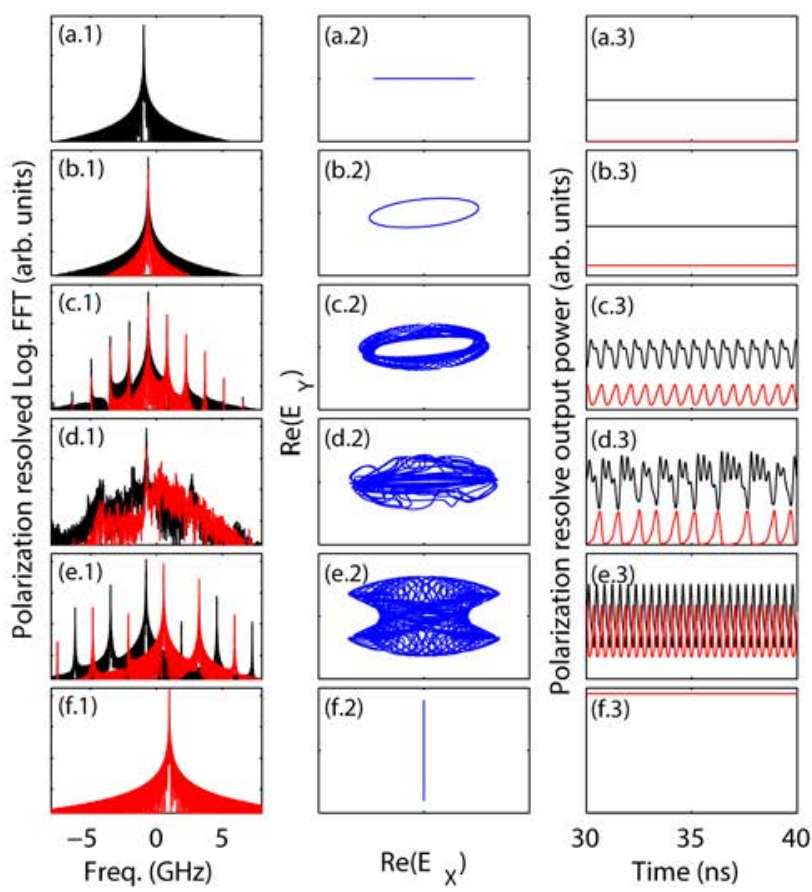

Figure 4. color online) Detailed characteristic of dynamical states for (a) $\mu=1.223$, (b) $\mu=1.323$, (c) $\mu=1.381$, (d) $\mu=1.416$, (e) $\mu=1.549$ and (f) $\mu=1.650$. The first column gives a logarithmic plot of the complex field FFT versus frequency, the second one is a representation of the output polarization polarization given by the system trajectory in the $\left(\operatorname{Re}\left(E_{X}\right), \operatorname{Re}\left(E_{Y}\right)\right)$ plane and the third gives the time traces observed for the dynamical states. Both the FFT and the time traces are polarization resolved, hence red and black plots are for the $\mathrm{X}$ and $\mathrm{Y}$ axis respectively.

and continuation ones, but more importantly they give complementary informations on bifurcations and on the dynamical behavior of the system.

The laser starts emitting in X-LP state with $E_{y}=0$, since the choice of the parameters leads to a smaller lasing threshold for the X-LP than for the Y-LP mode. As shown in figure 4-(a.1) the lasing frequency is slightly lower than $\omega_{0}$ and the laser emits in the LF mode. At $\mu=1.24$ the steady-state is destabilized by a pitchfork bifurcation, which can be clearly identified on figure 2, creating the two EP states. Figures 4-(b.1) and (b.2) show an elliptically polarized lasing mode with locking of the frequencies of the X-and Y-polarized modes.

When increasing the injection current further and starting from $\mu=1.36$, the EP states are both destabilized by a Hopf bifurcation creating two symmetrical limit cycles leading the system to a time-periodic self-pulsation (diamond symbol in figure 2). According to the continuation results of figure 2 (b) the frequency of the emerging time-periodic dynamics is about $1.5 \mathrm{GHz}$ which is close to $F_{\text {split }}$ and clearly less than $F_{R O}(\sim 3 \mathrm{GHz}$ in this injection current range). The limit cycle dynamics born on the EP solutions gets destabilized by a saddle-node bifurcation on limit cycle for a larger value of the injection current: $\mu=1.404$ (square symbol in figure 2). Figure 4 (c.1)-(c.3) shows an example of such limit cycle dynamics with an optical spectrum being centered at $\omega_{0}$ and with side-bands at harmonics of the fundamental frequency $\left(\sim F_{\text {split }}\right)$. The time-traces of the X- and Y-LP mode dynamics show anticorrelated dynamics of square-like waveforms. For a current value larger than the saddle-node bifurcation on limit cycle, the laser exhibits a chaotic dynamics that coexists with a stable steadystate solution with Y-linear polarization. The region of chaos extends up to $\mu \sim 1.47$, which corresponds to a saddle-node bifurcation on a time-periodic solution emerging from a subcritical Hopf bifurcation on the Y-LP 
mode (Hopf: diamond symbol on the red branch and saddle-node: square symbol on the orange branch in figure 2 ). The optical spectrum for the chaotic time-series is given in figure 4-(d.1) and it shows a broad spectrum with however spectral signatures at frequencies close to the one of now unstable limit cycle dynamics (green thin line on figure 2 around $1 \mathrm{GHz}$ ) and close to $F_{R O}$. The polarization resolved time-series of figure 4-(c.3) and (d.3) are strongly anti-correlated. As mentioned earlier, when increasing the current above $\mu=1.47$ the system reaches a new periodic solution born on the Y-LP steady-state branch from a subcritical Hopf bifurcation. An example of such dynamics is shown in Figure 4-(e.1) and (e.3). The laser exhibits a two mode self-pulsating dynamics. The optical spectra of the X and Y-LP modes show different central frequencies being separated by $F_{\text {split }}$ and with side-bands at frequencies being multiples of the frequency of the limit cycle dynamics, as determined by the continuation method (orange curve in figure 2). Interestingly, the polarization resolved time-series are not anticorrelated anymore, but the modes emit pulses in phase, see figure 4-(e.3). This limit cycle dynamics is stable up to a current value of about $\mu=1.638$, which corresponds to a second saddle-node bifurcation on the limit cycle solution. For larger current values the laser is left with a single attractor being a steady-state dynamics with Y-linear polarization, see Figure 4-(f.1)-(f.3).

If one cannot resolve the dynamics at the nanosecond time-scale, but rather looks only to the averaged optical power of the two LP components, one will see the polarization-resolved L-I curve of Figure 3 (c). The laser starts lasing with a X-LP mode and then a very gradual polarization switching takes place up to a current value of about $\mu=1.638$ where the laser stops lasing in the X-LP mode and is left with a single LP mode emission along the $\mathrm{Y}$ direction. It is worth mentioning that the fractional polarization in Figure 3 (a) is lower than one in the injection current range in between the Hopf bifurcation point on the EP solution and the saddle-node bifurcation on the limit cycle emerging from Y-LP steady-state, i.e. in the parameter range that corresponds to dynamical states of the light polarization.

For decreasing injection current the scenario is much more simple as the Y-LP is stable until $\mu \sim 1.32$. At this point the system jumps on one of the stable stationary EP state, and then the X-LP steady-state for current $\mu<1.24$. Therefore we have a large region of bistability between the Y-LP steady-state and the whole sequence of nonlinear dynamics.

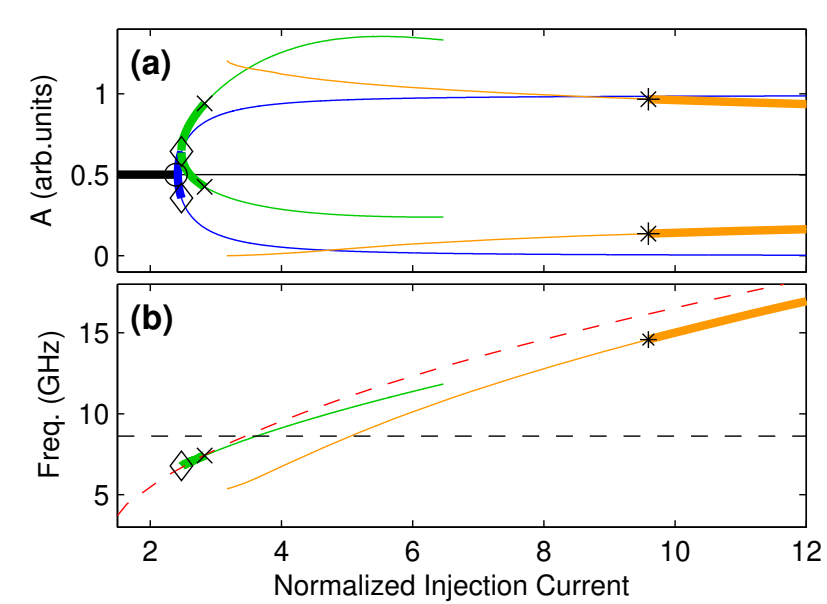

Figure 5. (color online) Continuation results for case 7 described in sec. 3 for $\gamma_{a}=-0.7$ and $\gamma_{p}=25$ : the stable (unstable) part of the branches are in thick (thin) lines. The caption is similar to the one of figure 2. We find a large region without any stable states and where the laser exhibits complex nonlinear dynamics.

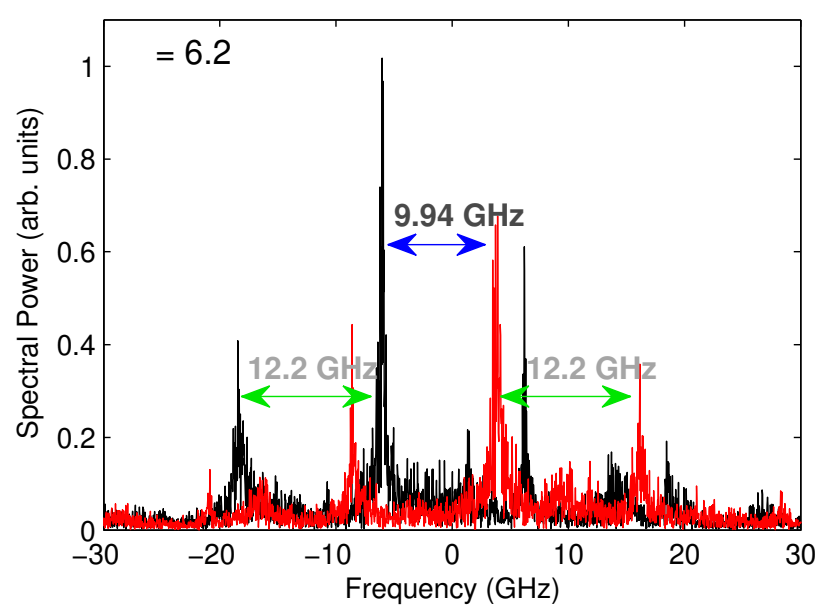

Figure 6. (color online) Optical spectrum for $\mu \sim 6.2$ for the same parameters as in figure 5 . We find a two-mode emission: the frequency splitting between the two mode is given by the unstable periodic solution born on Y-LP branch $F=9.94 \mathrm{GHz}$. We also find sidebands at the relaxation oscillation frequency $F_{R O}=12.2 \mathrm{GHz}$. 


\section{COMPARISON WITH EXPERIMENTAL RESULTS}

In the previous section we made a detail analysis of a type II PS which is in good qualitative agreement with the experiment of Sondermann et al. ${ }^{6}$ Experimentally the laser was found to go from the low to the high frequency state through a complex and dynamical transition: 1 / experiencing an elliptically polarized state, 2 / exhibiting self-pulsating dynamics at the birefringence induced frequency, $3 /$ emitting with a broad optical spectrum with two main peaks and multiple sidebands. In addition the reported experimental time-traces are close to the one described in figure 4-(d.3) even if correlation and DC-information are not available in the reported observations.

On the other hand we also paid a particular attention to the experimental report of Olejniczak et al ${ }^{8}$ describing a destabilization of the linearly polarized state without polarization switching; in particular a self-pulsing dynamic at the relaxation oscillation frequency is reported. Such behavior corresponds to scenario 7 , therefore we also made an in-depth analysis for the following parameters: $\gamma_{a}=-0.7$ and $\gamma_{p}=25$. The continuation results are displayed in figure 5 where we find a bifurcation sequence similar to the one for type II PS. However a chaotic region appears to be much larger and the periodic solution born on the Y-LP is stable only at very high injection current (orange line on figure 5). As shown in figure 5-(b) (the thick green line), the destabilization of the EP states leads the system to self-pulsing dynamics at the relaxation oscillation frequency $F_{R O}$. For higher current we only find complex nonlinear dynamics; a typical optical spectrum is given in figure 6 . We find a two-mode emission, one on each linear polarization, with sidebands at $F_{R O}$; the splitting frequency between the two modes is given by the frequency of the unstable periodic solution born on Y-LP (orange line on figure 5). A similar optical spectrum is reported in figure 6 of Ref. $^{8}$ where the splitting frequency of the two-modes is referred to as the "effective birefringence".

Those results demonstrate that we provide a convincing theoretical framework for the reported experimental observations. Indeed we find a good qualitative agreement in terms of bifurcation sequence, frequency and optical spectrum, but we also give new dynamical details about polarization switching which motivate additional experiments.

\section{CONCLUSION}

In summary, we made an in-depth analysis of the bifurcation scenarios leading a VCSEL to elliptically polarized emission and nonlinear polarization dynamics in the framework of the SFM model. We use an original combination of advance continuation techniques and direct numerical integration to demonstrate that the dynamics predicted by SFM are in fact a bifurcation problem. In section 3 we showed that many scenarios are predicted by the SFM model which involve various states and dynamics depending mainly on the anisotropy values. We also demonstrate that the frequency of the dynamics accompanying the polarization instabilities cannot be linked only to the characteristic frequency of the system (neither birefringence induced frequency nor the relaxation oscillation frequency) but has to be estimated from e.g. an analysis of the Hopf bifurcation as performed in this contribution. In section 4 we made a detailed investigation of a type II PS with a particular interest for the dynamical states experienced by the laser. We demonstrate a good agreement with the experimental observations of Sondermann et al.: ${ }^{6}$ the laser first experiences a transition through elliptically polarized state before entering a relatively large region of dynamics. In addition we provide new details on the dynamical states: we unveil two periodic solutions and highlight a large chaotic region influenced by the two unstable limit cycles. In the last section we reconcile two experiments with seemingly different conclusions on polarization switching dynamics, and demonstrate that they can be explained within a single theoretical framework: $1 /$ we summarized key experimental results of Ref., ${ }^{6}$ demonstrating the good agreement with theoretical results and $2 /$ we presented additional results for a bifurcation sequence in a good agreement with the observations of Olejniczak et al. ${ }^{8}$ Therefore our contribution not only brings new light on underlying mechanism of the dynamics accompanying the polarization switching events but also motivates new detailed experiment focusing on these rich dynamical transition. In the end, the complete understanding and mastering of these events may be very valuable for all optical processing and optical communications. 


\section{REFERENCES}

[1] Choquette, K. D., Richie, D. A., and Leibenguth, R. E., "Temperature dependence of gain-guided verticalcavity surface emitting laser polarization," Applied Physics Letters 64(16), 2062-2064 (1994).

[2] Choquette, K., Schneider, R., Lear, K., and Leibenguth, R., "Gain-dependent polarization properties of vertical-cavity lasers," Sel. Top. in Quantum Electron., IEEE Journal of 1, 661 -666 (jun 1995).

[3] Martín-Regalado, J., Miguel, M. S., Abraham, N. B., and Prati, F., "Polarization switching in quantum-well vertical-cavity surface-emitting lasers," Opt. Lett. 21, 351-353 (Mar 1996).

[4] van Exter, M. P., Willemsen, M. B., and Woerdman, J. P., "Polarization fluctuations in vertical-cavity semiconductor lasers," Phys. Rev. A 58, 4191-4205 (Nov 1998).

[5] Ackemann, T. and Sondermann, M., "Characteristics of polarization switching from the low to the high frequency mode in vertical-cavity surface-emitting lasers," Appl. Phys. Lett. 78, 3574 (2001).

[6] Sondermann, M., Ackemann, T., Balle, S., Mulet, J., and Panajotov, K., "Experimental and theoretical investigations on elliptically polarized dynamical transition states in the polarization switching of verticalcavity surface-emitting lasers," Optics Communications 235(46), 421 - 434 (2004).

[7] Olejniczak, L., Sciamanna, M., Thienpont, H., Panajotov, K., Mutig, A., Hopfer, F., and Bimberg, D., "Polarization switching in quantum-dot vertical-cavity surface-emitting lasers," Photonics Technology Letters, IEEE 21, 1008 -1010 (july15 2009).

[8] Olejniczak, L., Panajotov, K., Thienpont, H., Sciamanna, M., Mutig, A., Hopfer, F., and Bimberg, D., "Polarization switching and polarization mode hopping in quantum dot vertical-cavity surface-emitting lasers," Opt. Express 19, 2476-2484 (Jan 2011).

[9] Willemsen, M. B., Khalid, M. U. F., van Exter, M. P., and Woerdman, J. P., "Polarization switching of a vertical-cavity semiconductor laser as a kramers hopping problem," Phys. Rev. Lett. 82, 4815-4818 (Jun 1999).

[10] San Miguel, M., Feng, Q., and Moloney, J. V., "Light-polarization dynamics in surface-emitting semiconductor lasers," Phys. Rev. A 52, 1728 (Aug 1995).

[11] Martin-Regalado, J., Prati, F., San Miguel, M., and Abraham, N., "Polarization properties of vertical-cavity surface-emitting lasers," Quantum Electronics, IEEE Journal of 33, 765 -783 (may 1997).

[12] Travagnin, M., van Exter, M. P., Jansen van Doorn, A. K., and Woerdman, J. P., "Role of optical anisotropies in the polarization properties of surface-emitting semiconductor lasers," Phys. Rev. A 54, 1647-1660 (Aug 1996).

[13] Travagnin, M., "Linear anisotropies and polarization properties of vertical-cavity surface-emitting semiconductor lasers," Phys. Rev. A 56, 4094-4105 (Nov 1997).

[14] Erneux, T., Danckaert, J., Panajotov, K., and Veretennicoff, I., "Two-variable reduction of the san miguel $`$ feng $`$ moloney model for vertical-cavity surface-emitting lasers," Phys. Rev. A 59, 4660-4667 (Jun 1999).

[15] Travagnin, M., van Exter, M. P., and Woerdman, J. P., "Influence of carrier dynamics on the polarization stability and noise-induced polarization hopping in surface-emitting semiconductor lasers," Phys. Rev. A 56, 1497-1507 (Aug 1997).

[16] Prati, F., Caccia, P., and Castelli, F., "Effects of gain saturation on polarization switching in vertical-cavity surface-emitting lasers," Phys. Rev. A 66, 063811 (Dec 2002).

[17] Prati, F., Caccia, P., Bache, M., and Castelli, F., "Analysis of elliptically polarized states in vertical-cavitysurface-emitting lasers," Phys. Rev. A 69, 033810 (Mar 2004).

[18] Balle, S., Tolkachova, E., Miguel, M. S., Tredicce, J. R., Martín-Regalado, J., and Gahl, A., "Mechanisms of polarization switching in single-transverse-mode vertical-cavity surface-emitting lasers: thermal shift and nonlinear semiconductor dynamics," Opt. Lett. 24, 1121-1123 (Aug 1999).

[19] Van der Sande, G., Peeters, M., Veretennicoff, I., Danckaert, J., Verschaffelt, G., and Balle, S., "The effects of stress, temperature, and spin flips on polarization switching in vertical-cavity surface-emitting lasers," Quantum Electronics, IEEE Journal of 42, 898 -906 (sept. 2006).

[20] Engelborghs, K., Luzyanina, T., and Samaey, G., "Dde-biftool v. 2.00," tech. rep., TW-330, Dept. of CS, K. U. Leuven, Belgium (2002). 